\title{
Phenotypic adaptations of Leishmania donovani to recurrent miltefosine exposure and impact on sand fly infection
}

Sarah Hendrickx ${ }^{*}$, Lieselotte Van Bockstal ${ }^{1}$, Dimitri Bulté ${ }^{1}$, Annelies Mondelaers' ${ }^{1}$, Hamide Aslan', Luis Rivas², Louis Maes ${ }^{1}$ and Guy Caljon ${ }^{{ }^{*}}$ (D)

\begin{abstract}
Background: Since the introduction of miltefosine (MIL) as first-line therapy in the kala-azar elimination programme in the Indian subcontinent, treatment failure rates have been increasing. Since parasite infectivity and virulence may become altered upon treatment relapse, this laboratory study assessed the phenotypic effects of repeated in vitro and in vivo MIL exposure.
\end{abstract}

Methods: Syngeneic Leishmania donovani lines either or not exposed to MIL were compared for drug susceptibility, rate of promastigote multiplication and metacyclogenesis, macrophage infectivity and behaviour in the sand fly vector, Lutzomyia longipalpis.

Results: Promastigotes of both in vitro and in vivo MIL-selected strains displayed a slightly reduced drug susceptibility that was associated with a reduced MIL-accumulation linked to a lower copy number (disomic state) of chromosome 13 harboring the miltefosine transporter (LdMT) gene. In vitro selected promastigotes showed a lower rate of metacyclogenesis whereas the in vivo derived promastigotes displayed a moderately increased growth rate. Repeated MIL exposure did neither influence the parasite load nor metacyclogenesis in the sand fly vector.

Conclusions: Recurrent in vitro and in vivo MIL exposure evokes a number of very subtle phenotypic and genotypic changes which could make promastigotes less susceptible to MIL without attaining full resistance. These changes did not significantly impact on infection in the sand fly vector.

Keywords: Miltefosine, Leishmania, Fitness, Lutzomyia longipalpis, Drug uptake

\section{Background}

Although miltefosine (MIL) monotherapy was advocated about one decade ago as first-line treatment against visceral leishmaniasis (VL) in frame of the kala-azar elimination programme in the Indian subcontinent, its use has now largely been abandoned owing to the increase in therapeutic failures [1]. However, evidence for the

\footnotetext{
*Correspondence: Sarah.Hendrickx@uantwerpen.be; Guy.

Caljon@uantwerpen.be

${ }^{1}$ Laboratory of Microbiology, Parasitology and Hygiene (LMPH), University of Antwerp, Antwerp, Belgium

Full list of author information is available at the end of the article
}

involvement of drug resistance has remained scarce and the few reports of MIL resistance in clinical isolates were published only very recently [2]. The multifactorial causes of treatment failure are still not fully understood and involvement of the parasite's (epi-) phenotype has been suggested [3, 4]. Clinical isolates from relapse patients revealed alterations in parasite fitness rather than in drug susceptibility [3, 5]; however, the lack of large sets of paired pre- and post-treatment samples precludes direct comparison of syngeneic strains. Most laboratory studies circumvented this problem by repeatedly exposing promastigotes to MIL, hence generating resistant strains 
retaining an identical genetic background to the parent wild-type (WT). This way, refractoriness to MIL could be related to active drug efflux via LMDR1/LABCB4 or LABCG6 that are both P-glycoprotein-like transporters from the Leishmania ABC (ATP-binding cassette) family [6-9]. Later on, MIL resistance was linked to mutations in the putative L. donovani MIL transporter (LdMT) and/ or its $\beta$-subunit LdRos3, leading to a defective inward drug transport [10-12]. Although the broader impact of these mutations is still a topic of debate and may even be species-related [13, 14], MIL resistance definitely appears to be associated with alterations in parasite fitness. Although selection on promastigotes fairly rapidly resulted in resistance, repeated exposure of intracellular amastigotes both in vitro and in vivo did not result in reduced MIL susceptibility $[15,16]$. This may actually reflect the situation in the field where parasites recovered from patients retain an unaltered MIL susceptibility profile.

The present laboratory study used intracellular amastigotes of field isolates from Nepal that were repetitively exposed to MIL, both in vitro and in vivo in the hamster model. The phenotypic characteristics of the WT parent strain and derived MIL-exposed lines were comparatively evaluated to explore their impact on drug responsiveness, in vitro growth characteristics and transmission potential by the sand fly vector.

\section{Methods}

\section{Animals}

Female Swiss mice (20-25 g) and female golden hamsters $(80-100 \mathrm{~g})$ were obtained from Janvier (Le GenestSaint-Isle, France) and kept in quarantine for at least 5 days before infection. Food for laboratory rodents (Carfil, Arendonk, Belgium) and drinking water were available ad libitum. Hamsters were allocated to experimental units of 2 animals each.

\section{Leishmania parasites}

Intracellular amastigotes of the antimony $(\mathrm{Sb})$-resistant L. donovani clone MHOM/NP/03/BPK275/0cl18 were exposed in vitro to five successive treatment rounds of MIL (= BPK275/0cl18 MIL) [15]. Because of difficulties for its in vivo adaptation, another clonal line (= MHOM/ $\mathrm{NP} / 02 / \mathrm{BPK} 282 / 0 \mathrm{cl} 4)$ showing better infectivity in hamsters was used for the in vivo resistance selection experiments, as described elsewhere [16]. In brief, infection inoculates containing $2 \times 10^{7}$ spleen-derived amastigotes in phosphate-buffered saline (PBS) were administered by intracardial injection under isoflurane inhalation anesthesia. The general condition and body weight of the animals were monitored daily to evaluate the course of infection. Three weeks after infection, the animals were treated orally with MIL (20 mg/ml in PBS) for 5 days at $40 \mathrm{mg} / \mathrm{kg}$ single injection dose (s.i.d.) and followedup until clinical signs of relapse were noted (decrease in body weight, poor general appearance), hence enabling successive treatment-relapse cycles [16]. After five cycles, the resulting parasite population was harvested from the spleen and expanded in vitro as promastigotes (= BPK282/0cl4 MIL) in MIL-free medium. Both parent wild-type (WT) isolates had been obtained from bonemarrow aspirates of patients from the Terai endemic region in Nepal (BP Koirala Institute, Dharan, Nepal) within the frame of the EU-Kaladrug-R project and were provided by the Institute of Tropical Medicine (Antwerp, Belgium). Both strains were typed as $L$. donovani by cysteine proteinase $\mathrm{B}(\mathrm{CPB})-\mathrm{PCR}-\mathrm{RFLP}$ and their full genome sequences are available $[17,18]$. Promastigotes were cultured in HOMEM medium (Life technologies, Ghent, Belgium) supplemented with $10 \%$ inactivated fetal calf serum (iFCS).

\section{Drug formulations and preparation}

For the in vitro work, a stock solution of $20 \mathrm{mM}$ MIL $(\mathrm{MW}=407.57)$ (Carbosynth, Berkshire, UK) was prepared in PBS and stored at $4{ }^{\circ} \mathrm{C}$. For the in vitro uptake studies, fluorescent BODIPY-MIL analogue 2 was used [19]. For the treatment of animals, MIL was formulated in distilled water at $20 \mathrm{mg} / \mathrm{ml}$.

\section{Drug susceptibility determination}

Standard promastigote and intracellular amastigote assays were performed to assess MIL susceptibility. Primary peritoneal macrophages were harvested from starch-stimulated female Swiss mice. About $48 \mathrm{~h}$ after seeding, the cells were infected with stationary-phase promastigotes at a multiplicity of infection of 20:1. Twenty-four hours later, residual extracellular promastigotes were removed by washing and 2-fold MIL dilutions were subsequently added to the plates. After another 5 days of incubation at $37{ }^{\circ} \mathrm{C}$ and $5 \% \mathrm{CO}_{2}$, the plates were stained with Giemsa and $50 \%$ inhibitory concentrations $\left(\mathrm{IC}_{50}\right)$ were determined microscopically. To evaluate promastigote susceptibility, early log-phase promastigotes were exposed to two-fold MIL dilutions for $72 \mathrm{~h}$, after which $\mathrm{IC}_{50}$-values were determined upon addition of resazurin and fluorimetric reading [20].

\section{Evaluation of MIL uptake rates}

Intracellular accumulation of BODIPY-MIL [19] was compared between promastigote stages of the WT parent and the MIL-selected strains. Early log-phase (96 h) and stationary-phase $(168 \mathrm{~h})$ promastigotes were suspended in RPMI-1640 medium without phenol red at a concentration of $5 \times 10^{6}$ cells $/ \mathrm{ml}$ and were labeled with 
$0.5 \mu \mathrm{M}$ BODIPY-MIL in the dark for $1 \mathrm{~h}$ at room temperature. Upon incubation, the non-internalized drug was removed by washing the parasites twice with icecold PBS containing 1\% bovine serum albumin, resuspended in RPMI-1640 medium and kept on ice to block outward drug transport. Quantification of intracellular MIL accumulation was achieved by measuring fluorescence intensity in the FL2-channel by flow cytometry (FACSCalibur $^{\circledR}$ ). TO-PRO ${ }^{\circledR}-3$ iodide (Molecular Probes ${ }^{\circledR}$, Eugene, OR, USA) was used for live/dead staining and 100,000 events per sample were recorded [21]. Nontreated parasites were included as negative control. Data analysis was performed using BD CellquestPro ${ }^{\circledR}$ software.

\section{Parasite fitness analysis Promastigote growth}

Promastigote growth curves were made [22] to compare the in vitro growth of the WT parent and MIL-exposed derived strains. After passage through fine needles (21

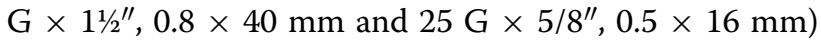
to break clustering, the promastigotes were diluted in PBS and counted by flow cytometry. Exactly $5 \times 10^{5} \log$ phase promastigotes/ml were seeded in $5 \mathrm{ml}$ HOMEM and their number was determined flow cytometrically (FACSCalibur $^{\circledR}$ ) every $24 \mathrm{~h}$ for a total of 10 days. Three independent repeats of each strain were run in parallel and growth curves were made by plotting the average parasite density \pm the standard error of the mean (SE) over time.

\section{Morphological evaluation of metacyclogenesis}

To evaluate metacyclogenesis at each time point, the flagellum/cell body length ratio was determined. Promastigotes were air-dried on a glass microscopy slide, fixed with methanol, Giemsa-stained and analyzed with bright field microscopy (Axiovert $200 \mathrm{~m}^{\circledR}$; Carl Zeiss, Zaventem, Belgium) using Zeiss Axiocam MRm ${ }^{\circledR}$. The flagellum and cell body lengths of at least 50 parasites per slide were determined using the Axiovision ${ }^{\circledR}$ software and the percentage of metacyclics present in each sample was calculated. Parasites were considered metacyclic when the flagellum/cell body ratio exceeded 2 [22].

\section{Promastigote infectivity}

The in vitro infectivity of WT parent and MIL-exposed promastigotes was compared by infecting primary peritoneal mouse macrophages at a 20/1 parasite/macrophage ratio and subsequent microscopic assessment of the infection index (total number of amastigotes counted / total number of cells counted) upon Giemsastaining $24 \mathrm{~h}$ after infection. Promastigote viability was determined flow cytometrically before infection with the single-stain viability dye $\mathrm{TO}-\mathrm{PRO}^{\circledR}-3$ iodide $(10 \mu \mathrm{M})$
(Molecular Probes ${ }^{\circledR}$ ) to ascertain identical infection inoculates for each strain, hereby ensuring full comparability. The percentage of infected cells was calculated to determine the cell infectivity.

\section{Promastigote multiplication and metacyclogenesis in the sand fly vector}

The effect of repeated MIL exposure on overall parasite fitness in the sand fly vector was evaluated in a Lutzomyia longipalpis colony maintained under standard conditions [23]. To simulate natural transmission, female sand flies (3-5 days-old) were fed with heat-inactivated mouse blood containing $5 \times 10^{6}$ promastigotes $/ \mathrm{ml}$ through a blood feeding device covered with a chicken-skin membrane. After $24 \mathrm{~h}$, blood-fed females were isolated and further maintained at $26{ }^{\circ} \mathrm{C}$. To follow-up the infection over time, the flies were dissected on 2, 5, 7 and 9 days post-infection (dpi) to microscopically check the presence and localization of promastigotes in their guts, or by crushing the total gut content in PBS and microscopically quantifying the infection intensity using a KOVA counting chamber. Metacyclogenesis was evaluated morphometrically by light microscopy in parallel. The experimental infections were performed with an independent repeat for each strain. Parasites were also isolated from the vector on $7 \mathrm{dpi}$ and cultivated in HOMEM promastigote medium supplemented with $5 \%$ penicillin-streptomycin. When sufficiently dense log-phase cultures were obtained, post-fly promastigotes and intracellular amastigotes were used for routine MIL susceptibility determination, as described above.

\section{MIL transporter copy number comparison by qPCR}

Given the known plasticity in chromosome copy number for chromosome 13 (harboring the LdMT MILtransporter gene) and chromosome 32 (harboring the LdROS3-subunit gene), the copy number of both was determined by qPCR. Chromosomes with stable somy (and preferentially large size) were selected as references based on previous research, i.e. chromosome 24 (stably disomic), chromosome 31 (mostly tetrasomic) and chromosome 36 (stably disomic) [24].

Upon selection of reference genes based on gene lists for annotated chromosomes (https://www.ncbi. nlm.nih.gov/genome/?term=leishmania+donovani), the single copy nature of these genes was confirmed by BLAST in the TriTryp database and conservation of the gene sequence was checked in $L$. donovani (BPK282A1) and L. infantum (JPCM5). Primers were designed to amplify a 116-bp region in chromosome 24 , a 64-bp region in chromosome 31 and a 96-bp region in chromosome 36 by using online software (http:// www.ncbi.nlm.nih.gov/tools/primer-blast/) and were 
synthesized by Integrated DNA Technologies (IDT, Belgium). DNA extraction of promastigote cultures was performed using the QIAamp DNA Mini and Blood Mini Kit (Qiagen, Hilden, Germany) according to the manufacturer's instructions. Resulting DNA concentrations and purities were estimated by NanoDrop2000c spectrophotometer (Thermo Fisher Scientific, Merelbeke, Belgium). The Step One Plus real-time PCR system (Applied Biosystems, Bleiswijk, the Netherlands) was used for all PCR assays and melt curve analyses. Each assay was run according to the conditions stated in Additional file 1: Table S1 in technical duplicate. Resulting Cq-values were used to determine the corresponding chromosome copy numbers. The normalized relative quantity of chromosome 13 (containing $L d M T$ ) and chromosome 32 (containing LdROS3) was calculated using Qbase software version 3.2 and using chromosomes 24,31 and 36 as references.

\section{Statistical analysis}

All statistical analyses were performed using Graphpad Prism version 6.00 software. Statistical differences between WT parent and MIL-exposed derived strains and between the different time points within one group were determined using 2-way ANOVA with Bonferroni post-hoc comparisons for parasite growth, parasite morphology and infection indices. Morphological and infection indices inter-group comparisons were carried out using the non-parametric Friedman test followed by Dunn's post-hoc comparisons. Statistical comparisons of relative copy numbers and sand fly parasite load and percentage of metacyclics were made using the Student's t-test. Tests were considered statistically significant if $P<0.05$.

\section{Results}

\section{Drug susceptibility determination}

Repeated MIL exposure of $L$. donovani amastigotes in vitro (BPK275/0-cl18 MIL) or in vivo (BPK282/0-cl4
MIL) did not result in a significant increase in intracellular amastigote $\mathrm{IC}_{50}$ in the in vitro drug susceptibility assay (Table 1). The marginal decrease in promastigote susceptibility following both in vitro and in vivo selection was insufficient to account for full MIL resistance [25]. To evaluate the stability of this 'altered' promastigote phenotype upon passage in the sand fly, MIL susceptibility was determined on 'post-fly' derived promastigotes with negligible differences in susceptibility between the different 'post-fly' subcultures. After passage in the sand fly, MIL IC 50 values show an overall trend to be lower in both promastigotes and amastigotes (Table 1).

\section{MIL transporter gene copy number determination by qPCR}

To check the involvement of aneuploidy, the relative abundance of the $L d M T$ gene (chromosome 13) and the $\beta$-subunit gene LdROS3 (chromosome 32) were compared between WT and MIL-selected parasites before and after passage in the sand fly. A decrease in $L d M T$ copy number was noted after MIL selection for both strains (Fig. 1) and is compatible with a decrease in chromosome ploidy from trisomic to disomic state $\left(\mathrm{BPK} 275: F_{(3,30)}=9.861, P=0.0001 ; \mathrm{BPK} 282: F_{(3,16)}=\right.$ $13.12, P=0.0001)$. BODIPY-MIL uptake studies demonstrated significantly decreased drug accumulation in $\log$-phase promastigotes ( $96 \mathrm{~h}$ in culture) of both in vitro and in vivo MIL-selected parasite strains, and in stationary-phase (168 h in culture) of the in vivo MILselected strain (Fig. 2) (BPK275: $F_{(1,4)}=4.155, P=$ 0.1111; BPK282: $\left.F_{(1,2)}=6.043, P=0.1332\right)$. After sand fly passage, the $L d M T$ gene copy number increased for the WT (Fig. 1), making it more susceptible to MIL than before passage through the vector (Table 1). The copy number did not significantly change in the MILselected strains isolated from sand flies.

\section{Parasite fitness analysis Promastigote growth}

Promastigote multiplication revealed no biologically significant variations between the WT parent and the

Table 1 Drug susceptibility of the wild-type (WT) parent and the derived isolates exposed for five cycles to MIL

\begin{tabular}{|c|c|c|c|c|}
\hline Strain & $\begin{array}{l}\text { Intracellular amastigote } \mathrm{IC}_{50} \pm \\
\mathrm{SE}(\mu \mathrm{M})\end{array}$ & $\begin{array}{l}\text { Post-fly amastigote } \mathrm{IC}_{50} \pm \mathrm{SE} \\
(\mu \mathrm{M})\end{array}$ & Promastigote $\mathrm{IC}_{50} \pm \mathrm{SE}(\mu \mathrm{M})$ & $\begin{array}{l}\text { Post-fly } \\
\text { promastigote } \mathrm{IC}_{50} \\
\pm \mathrm{SE}(\mu \mathrm{M})\end{array}$ \\
\hline BPK275/0-cl18WT & $2.4 \pm 0.3$ & $0.4 \pm 0.1$ & $5.8 \pm 1.0$ & $2.8 \pm 0.3$ \\
\hline BPK275/0-cl18 MIL & $3.1 \pm 0.4$ & $1.4 \pm 0.1$ & $9.8 \pm 1.2$ & $4.5 \pm 0.4$ \\
\hline BPK282/0-cl4 WT & $2.4 \pm 0.4$ & $0.6 \pm 0.1$ & $2.6 \pm 0.8$ & $5.0 \pm 0.6$ \\
\hline BPK282/0-Cl4 MIL & $1.2 \pm 0.1$ & $1.0 \pm 0.2$ & $9.7 \pm 2.5$ & $7.2 \pm 1.3$ \\
\hline
\end{tabular}

Notes: Susceptibility of the different strains is expressed as the average $50 \%$ inhibitory concentration $\left(\mathrm{IC}_{50}\right) \pm$ standard error of the mean (SE) and is the result of at least four independent experiments run in duplicate 


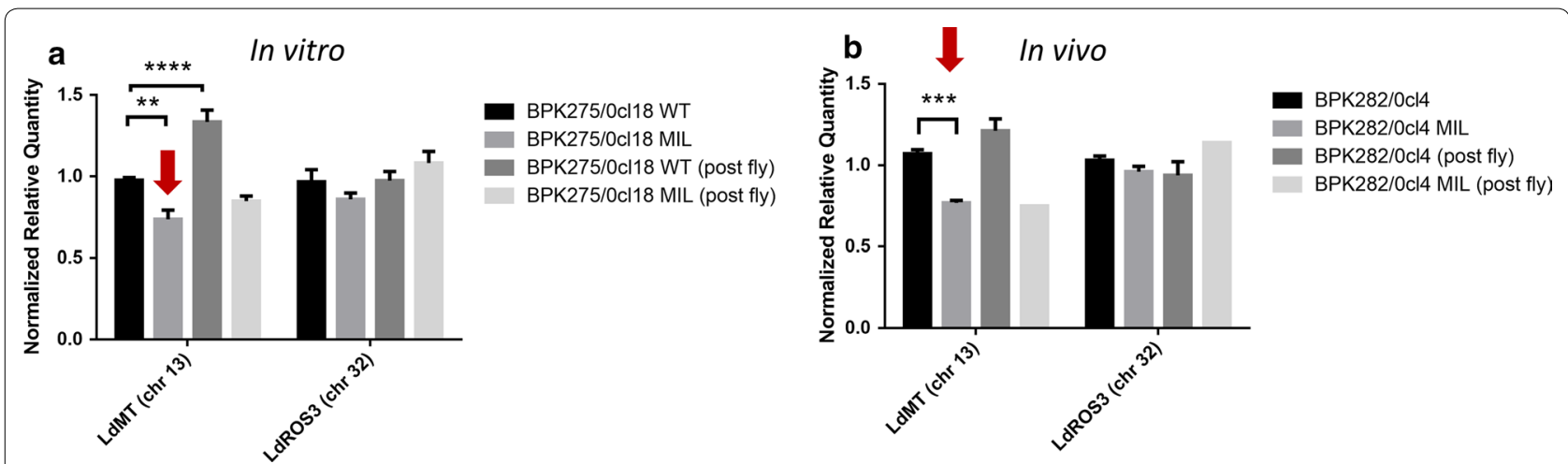

Fig. 1 Comparison of the normalized relative quantity of $L d M T$ (chromosome 13) and LdROS3 (chromosome 32) for BPK275/0Cl18 (a) and BPK282/0cl4 (b). A significant reduction in LdMT relative quantity could be detected between the WT and the in vitro and in vivo MIL-selected lines, and is compatible with WT being trisomic and the MIL-selected line disomic for chromosome 13 (arrows). A significant difference in LdMT could also be noted for the WT strain before and after passage in the sand fly. No significant differences could be detected in LdROS3 gene copy number for the in vivo-selected. Results are based on duplicate measurements of at least three biological replicates and are expressed as the mean ( \pm SE) relative quantity normalized using chromosome 24 (stably disomic), chromosome 31 (mostly tetrasomic) and chromosome 36 (stably disomic) as references. ${ }^{* *} P<0.01,{ }^{* * *} P<0.001,{ }^{* * *} P<0.0001$

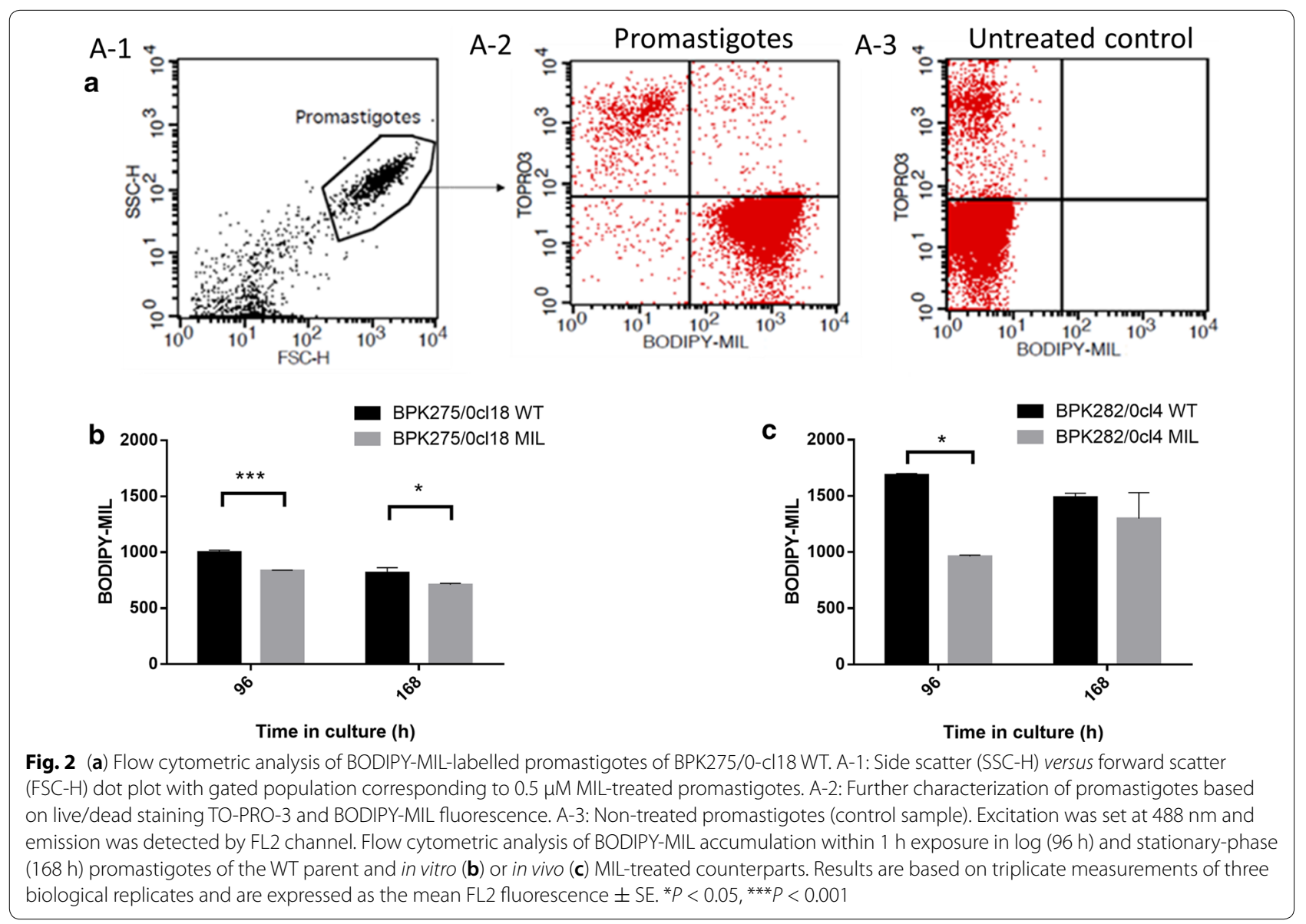



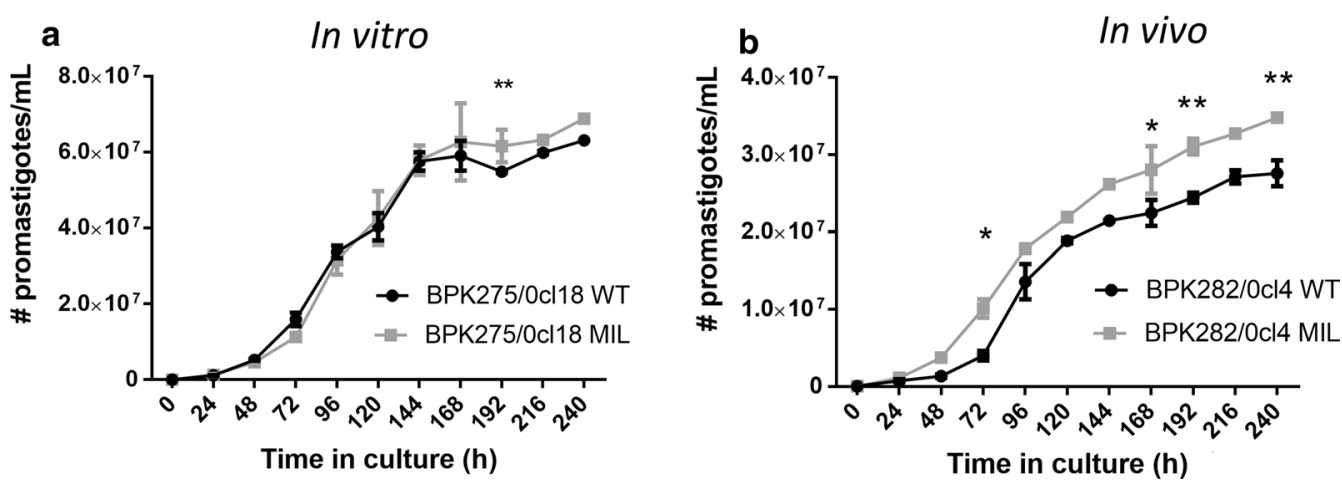

Fig. 3 Promastigote growth curves of wild-type parent (WT) and derived counterparts that were repeatedly exposed to MIL, either in vitro (a) or in vivo (b). No biologically significant differences could be observed for the in vitro MIL-exposed strain (a) whereas successive selection cycles in vivo resulted in parasites with a significantly increased in vitro promastigote growth (b). Results are expressed as the mean number of parasites in culture $\pm \mathrm{SE}$ and are based on three independent replicates. ${ }^{*} P<0.05,{ }^{* *} P<0.01$

in vitro MIL-exposed counterpart (Fig. 3a). In vivo MIL exposure resulted in parasites with a significantly increased promastigote multiplication rate compared to its WT parent strain (Fig. 3b) $\left(F_{(10,64)}=263.4, P<\right.$ 0.0001).

\section{Morphological evaluation of metacyclogenesis}

Promastigote transformation into metacyclics (flagellum length/cell body ratio $>2$ ) was affected after recurrent MIL treatment in vitro, whereas no impact of in vivo treatment could be demonstrated. Phenotypic differences upon in vitro selection were observed as an altered morphometric profile at the population level with a lower percentage $(\max 40 \%)$ of metacyclic promastigotes versus $80 \%$ in the WT population (Fig. 4$)\left(\right.$ BPK275: $F_{(5,517)}=$ 3.525, $P=0.0038$; BPK282: $\left.F_{(3,388)}=0.4365, P=0.7270\right)$.

\section{Promastigote infectivity in macrophages}

The highest infection ratios were reached when cells were infected with promastigotes from stationary-phase cultures in which metacyclics were most abundant. No effect was detected of both in vitro and in vivo MIL exposure on promastigote infectivity (Fig. 5) (BPK275: $F_{(3,8)}=$ $0.9287, P=4701$; BPK282: $\left.F_{(4,10)}=0.6246, P=0.6556\right)$.

\section{Promastigote multiplication and metacyclogenesis in the sand fly vector}

To compare the transmission potential, parasite survival and multiplication were evaluated in the L. longipalpis sand fly vector. When the total parasite load per gut and the percentage of metacyclic promastigotes were compared, no significant differences could be observed between the WT parent strains and the derived lines that were repeatedly exposed to MIL either in vitro (BPK275) or in vivo (BPK282) (Fig. 6) (BPK275: $F_{(8,81)}=0.1893, P$ $=0.9918$; BPK282: $\left.F_{(3,42)}=0.2239, P=0.8793\right)$.

\section{Discussion}

The growing number of MIL relapse cases in the Indian subcontinent is severely compromising the further use of MIL in monotherapy. Even though treatment failures have become fairly common, the exact causal reasons still remain to be elucidated. For example, involvement of host-related parameters linking treatment failure and post-treatment relapse to drug pharmacokinetics and dynamics (PK/PD) have already been indicated [26, 27] as well as the link of MIL relapse to both drug-related and parasite-related factors [28]. It was shown that a MIL-resistant phenotype can impact on parasite fitness $[13,14,29]$ and that clinical MIL relapse may be associated with enhanced infectivity [3]. The present laboratory study specifically aimed to evaluate the phenotypic impact of repeated MIL exposure using syngeneic strains obtained through both in vitro and in vivo selection procedures $[15,16]$.

Given the sometimes difficult adaptation of clinical isolates to the hamster model, two reference strains from an EU-FP7 Programme (Kaladrug-R project 222895) were used in this study. Both were selected for their background susceptibility to antimony (Sb), with BPK275/0/cl18 having a Sb-resistant background and BPK282/0-cl4 having a Sb-susceptible background. Although a Sbresistance background may alter the interaction with several drugs [30], it does not impact on the experimental selection for MIL resistance [31], hence allowing the use of both strains without challenging our stated research aims. The intrinsically low susceptibility of BPK282/0-cl4 towards paromomycin, a feature that has been associated with increased infectivity, could possibly explain its relatively more easy adaptation to the hamster model [22].

Despite former reports on the rapid and straightforeward selection of MIL-resistance in promastigotes [31-33], successive selection cycles on intracellular 


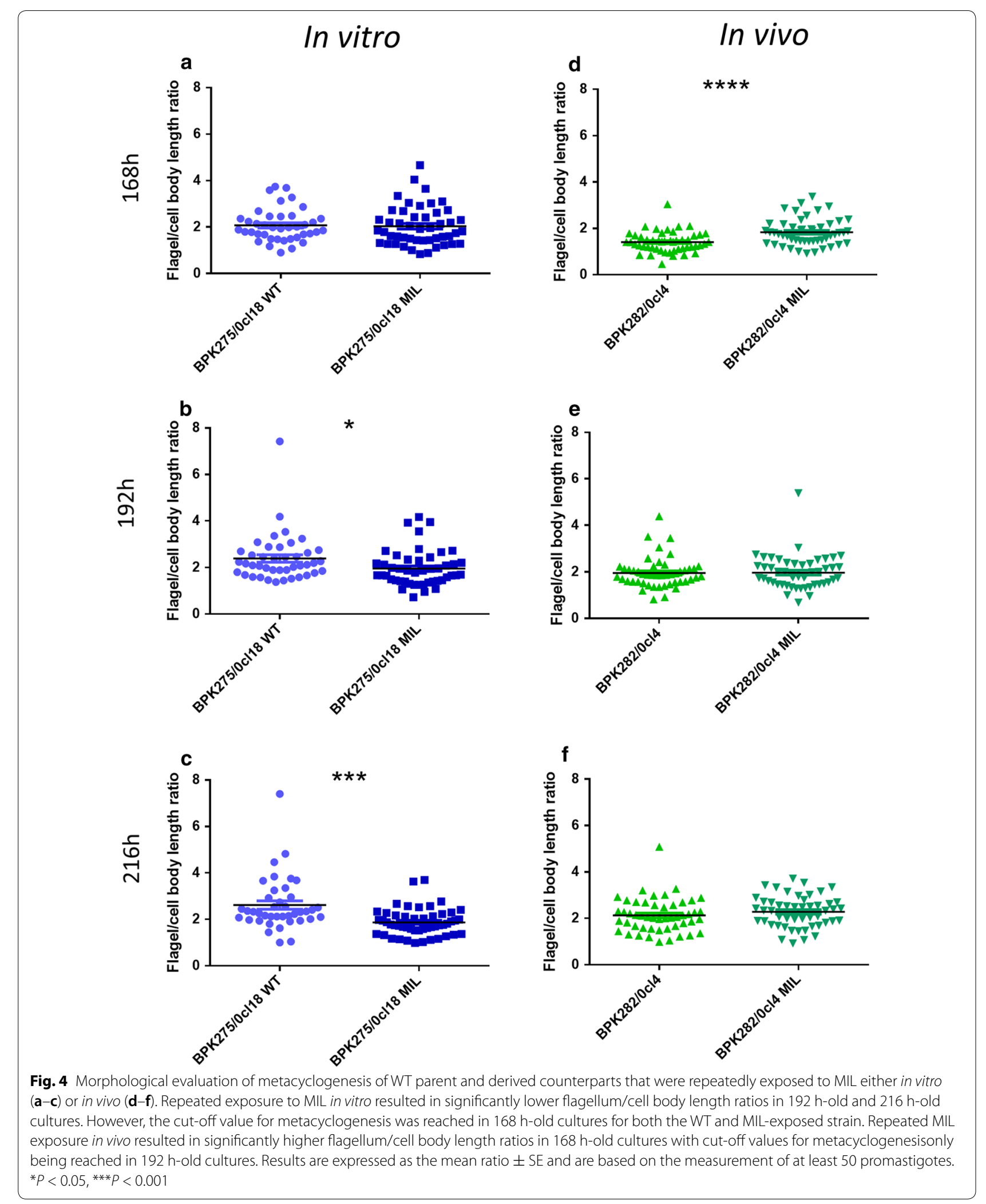




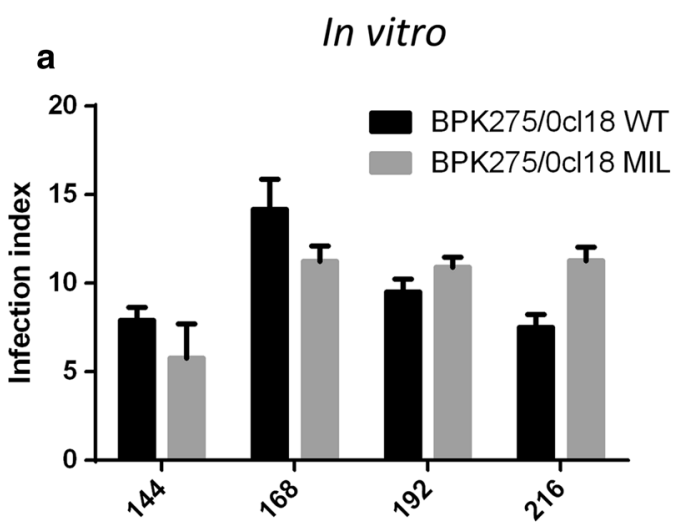

Time in culture $(\mathbf{h})$

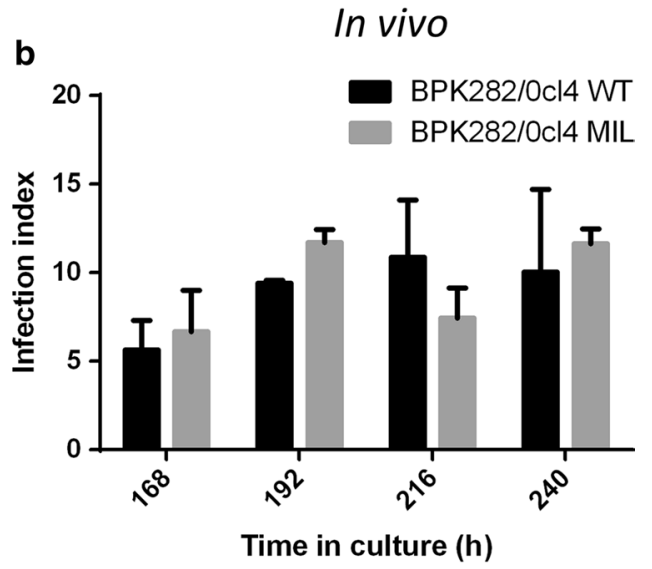

Fig. 5 Promastigote infectivity of both WT parent and derived counterparts that were repeatedly exposed to MIL either in vitro (a) or in vivo (b). No significant differences in in vitro macrophage infectivity are observed. Maximum infectivity was reached with 168 h-old cultures for BPK275/0-cl18 and with $192 \mathrm{~h} / 216 \mathrm{~h}$-old cultures for BPK282/0-cl4. Results are expressed as the mean infection index $\pm \mathrm{SE}$ and are based on three independent replicates

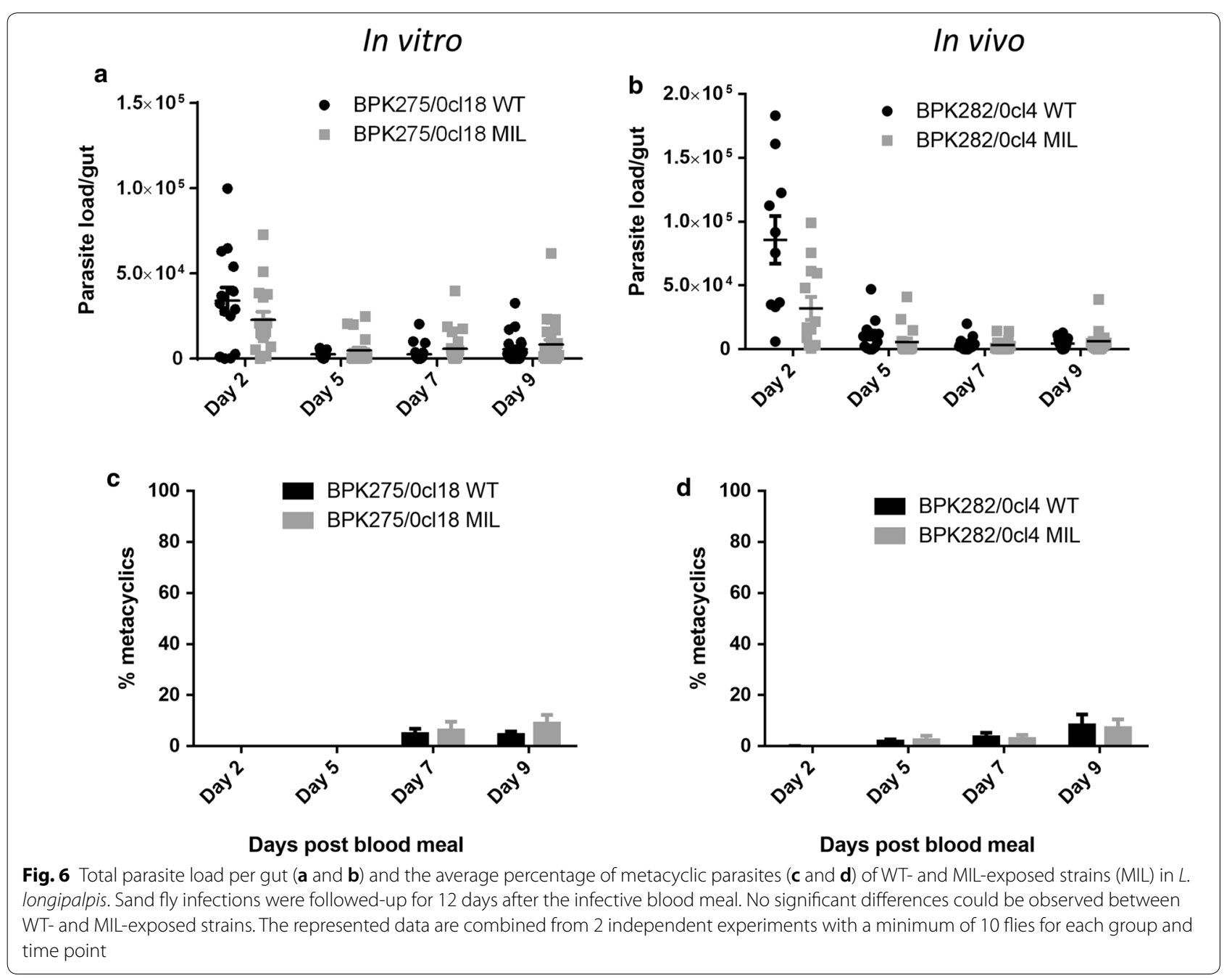


amastigotes both in vitro and in vivo failed to result in a drug-resistant phenotype [15, 34], creating the possibility to assess the impact of repeated MIL exposure without the alterations in parasite fitness as observed with full MIL resistance [13, 14, 29]. In the present study, recurrent in vitro and in vivo MIL exposure only resulted in a marginal decrease in promastigote susceptibility, which corroborates other studies describing the existence of an 'intermediate' resistant phenotype characterized by a partial decrease in susceptibility as a step towards full resistance [31]. Post-treatment relapse in the field has been linked to only a minor decrease in MIL susceptibility, with this 'intermediate' resistant phenotype behaving similarly to the non-exposed wild-type strains [35]. This contrasts with other studies describing an increased infectivity for relapse-derived strains $[3,5]$ which is actually unexpected given the detrimental changes in fitness upon aqcuisition of full resistance [13, 14, 29].

A functional LdMT transporter has been shown to be imperative for intrinsic MIL susceptibility. In the 'intermediate' resistant phenotype, biochemical analyses revealed a moderate but significant reduction of MIL accumulation. Since efflux rates are not significantly increased (Additional file 1: Figure S1), outward drug transporters are likely not primarily involved in the observed decrease in MIL susceptibility. Similarly, clinical isolates with an increased tolerance to MIL have been reported to show a decreased drug uptake [5]. The lower steady-state MIL accumulation correlates with a lower $L d M T$ gene copy number that probably arose from the parasite's extreme genomic plasticity under stress conditions [36]. Specifically, the modified $L d M T$ gene dosage could result from a decreased somy of chromosome 13 $[31,37]$. Indeed, a qPCR-based analysis showed a significant decrease in normalized relative quantity of this chromosome for both strains after repeated MIL-exposure and is compatible with a change from a trisomic to disomic state.

As former research already demonstrated the possibility of emerging phenotypic heterogeneity [38, 39], the present study was carried out using the polyclonal population obtained after the repeated drug exposure. As a result of its genomic plasticity, the parasite can create phenotypic diversity by mosaic aneuploidy, i.e. varying chromosomal content between cells leading to intrastrain genomic heterogeneity [36]. The observed change from a trisomic to disomic state of chromosome 13 after repeated in vitro MIL exposure aligns with the intermediate' resistance on promastigote level. Corroborating a previous study, the moderately increased promastigote $\mathrm{IC}_{50}$ does not translate into susceptibility changes at the amastigote level [31]. Next to drug susceptibility, MIL-exposed parasites exhibited slightly altered growth and/or metacyclogenesis phenotypes under in vitro culture conditions. Despite these changes, parasite infectivity and metacyclogenesis remained unaltered in the sand fly vector: MIL-exposed lines appeared to retain a disomic state of chromosome 13 after sand fly passage. Recent research using polyclonal BPK275 demonstrated a swift adaptation to a disomic state of chromosome 13 upon passage in the sand fly [24]. This implies that the 'intermediate' resistant phenotype would be stable, as we indeed observed the disomic state of chromosome 13 to be conserved after sand fly passage. Nevertheless, absolute $\mathrm{IC}_{50}$ values seemed to be slightly lower following passage through the sand fly vector, but these could not be attributed to significant changes in $L d M T$ or $L d R o s 3$ gene dosage.

Collectively, our observations indicate that recurrent MIL exposure evokes a range of subtle phenotypic changes, without significant impact on sand fly infection and development of infective parasite stages.

\section{Conclusions}

Repeated in vitro and in vivo exposure of Leishmania parasites to MIL evokes a number of subtle phenotypic changes in promastigotes, including a slightly reduced MIL susceptibility. This was found to be associated with a reduced $L d M T$ gene copy number and reduced drug accumulation. Despite these phenotypic changes, parasite infectivity and metacyclogenesis remained unaltered inside the sand fly vector.

\section{Supplementary information}

Supplementary information accompanies this paper at https://doi. org/10.1186/s13071-020-3972-z.

Additional file 1: Text S1. Methods. Table S1. Primer sequences and qPCR settings used to evaluate the MIL transporter copy number by qPCR. Text S2. Results. Figure S1. Time-dependent efflux of BODIPY-MIL. Delta FL2/min was calculated for log-phase (96 h) and stationary-phase (168 h) promastigotes of WT and MIL-exposed strains. Efflux rates were not significantly altered. Results are based on triplicate measurements of three biological replicates and are expressed as the average of delta FL2/ $\min \pm \mathrm{SE}$.

\section{Abbreviations}

dpi: Days post-infection; $I_{50}$ : Inhibitory concentration 50\%; LdMT: Leishmania donovani miltefosine transporter; MIL: Miltefosine; PBS: Phosphate-buffered saline; Sb: Antimony; SE: Standard error of the mean; s.i.d: Single injection dose; VL: Visceral leishmaniasis; WT: Wild-type.

\section{Acknowledgements}

The authors wish to thank Pim-Bart Feijens, Mandy Vermont and Rik Hendrickx for their excellent technical assistance with the laboratory and animal work. The authors thank the clinicians at the B. P. Koirala Institute of Health Sciences in Dharan, Nepal for providing the L. donovani patient isolates, conducted in frame of the EU-FP7 Programme (Kaladrug-R project, Grant 222895). The authors also want to acknowledge Shaden Kamhawi, Jesus Valenzuela and Claudio Meneses (National Institutes of Health, USA) for their help in establishing the L. longipalpis colony at the University of Antwerp. LMPH participates 
in COST Action CM1307 (targeted chemotherapy towards diseases caused by endoparasites) and is a partner of the Antwerp Drug Discovery Network (ADDN, http://www.addn.be) and the Excellence Centre "Infla-Med" (http:// www.uantwerpen.be/infla-med)

\section{Authors' contributions}

$\mathrm{SH}$, LM and GC conceptualized the study. SH, AM and DB executed and analyzed all in vitro experiments. SH, LVB and HA performed the sand fly experiments. LR kindly provided the BODIPY-MIL. SH, LM and GC wrote the manuscript. All authors read and approved the final manuscript.

\section{Funding}

Funding was available through the Research Fund Flanders (G051812N, $1210317 \mathrm{~N}$ and $1189617 \mathrm{~N}$ ) and a research fund of the University of Antwerp (TT-ZAPBOF 33049 and TOP-BOF 35017). LR was supported by Redes de Investigación Cooperativa RETICS-FEDER RD16/0027/0010.

\section{Availability of data and materials}

All data generated or analyzed during this study are included in this published article. The raw datasets are available from the corresponding author upon reasonable request.

\section{Ethics approval and consent to participate}

The use of laboratory rodents was carried out in accordance to all mandatory guidelines (EU directives, including the Revised Directive 2010/63/EU on the Protection of Animals used for Scientific Purposes that came into force on $01 / 01 / 2013$, and the declaration of Helsinki in its latest version) and was approved by the ethical committee of the University of Antwerp, Belgium [UAECD 2011-77 (17-02-2012)].

\section{Consent for publication}

Not applicable.

\section{Competing interests}

The authors declare that they have no competing interests.

\section{Author details}

${ }^{1}$ Laboratory of Microbiology, Parasitology and Hygiene (LMPH), University of Antwerp, Antwerp, Belgium. ${ }^{2}$ Centro de investigaciones Biológicas - CSIC, Madrid, Spain

Received: 24 January 2020 Accepted: 14 February 2020

Published online: 22 February 2020

\section{References}

1. Rijal S, Ostyn B, Uranw S, Rai K, Bhattarai NR, Dorlo TP, et al. Increasing failure of miltefosine in the treatment of kala-azar in Nepal and the potential role of parasite drug resistance, reinfection, or noncompliance. Clin Infect Dis. 2013;56:1530-8.

2. Srivastava S, Mishra J, Gupta AK, Singh A, Shankar P, Singh S. Laboratory confirmed miltefosine resistant cases of visceral leishmaniasis from India. Parasites Vectors. 2017;10:49.

3. Rai K, Cuypers B, Bhattarai NR, Uranw S, Berg M, Ostyn B, et al. Relapse after treatment with miltefosine for visceral leishmaniasis is associated with increased infectivity of the infecting Leishmania donovani strain. MBio. 2013;4:e00611-3.

4. Vanaerschot M, Dumetz F, Roy S, Ponte-Sucre A, Arevalo J, Dujardin JC. Treatment failure in leishmaniasis: drug-resistance or another (epi-) phenotype? Expert Rev Anti Infect Ther. 2014;12:937-46.

5. Deep DK, Singh R, Bhandari V, Verma A, Sharma V, Wajid S, et al. Increased miltefosine tolerance in clinical isolates of Leishmania donovani is associated with reduced drug accumulation, increased infectivity and resistance to oxidative stress. PLoS Negl Trop Dis. 2017:11:e0005641.

6. Perez-Victoria JM, Cortes-Selva F, Parodi-Talice A, Bavchvarov BI, Perez-Victoria FJ, Munoz-Martinez F, et al. Combination of suboptimal doses of inhibitors targeting different domains of LtrMDR1 efficiently overcomes resistance of Leishmania spp. to miltefosine by inhibiting drug efflux. Antimicrob Agents Chemother. 2006;50:3102-10.

7. Castanys-Munoz E, Alder-Baerens N, Pomorski T, Gamarro F, Castanys S. A novel ATP-binding cassette transporter from Leishmania is involved in transport of phosphatidylcholine analogues and resistance to alkylphospholipids. Mol Microbiol. 2007;64:1141-53.

8. Castanys-Munoz E, Perez-Victoria JM, Gamarro F, Castanys S. Characterization of an ABCG-like transporter from the protozoan parasite Leishmania with a role in drug resistance and transbilayer lipid movement. Antimicrob Agents Chemother. 2008;52:3573-9.

9. BoseDasgupta S, Ganguly A, Roy A, Mukherjee T, Majumder HK. A novel ATP-binding cassette transporter, ABCG6 is involved in chemoresistance of Leishmania. Mol Biochem Parasitol. 2008;158:176-88.

10. Seifert K, Perez-Victoria FJ, Stettler M, Sanchez-Canete MP, Castanys S, Gamarro F, et al. Inactivation of the miltefosine transporter, LdMT, causes miltefosine resistance that is conferred to the amastigote stage of Leishmania donovani and persists in vivo. Int J Antimicrob Agents. 2007:30:229-35.

11. Perez-Victoria FJ, Castanys S, Gamarro F. Leishmania donovani resistance to miltefosine involves a defective inward translocation of the drug. Antimicrob Agents Chemother. 2003;47:2397-403.

12. Mondelaers A, Sanchez-Canete MP, Hendrickx S, Eberhardt E, GarciaHernandez R, Lachaud L, et al. Genomic and molecular characterization of miltefosine resistance in Leishmania infantum strains with either natural or acquired resistance through experimental selection of intracellular amastigotes. PLoS ONE. 2016;11:e0154101.

13. Turner KG, Vacchina P, Robles-Murguia M, Wadsworth M, McDowell MA Morales MA. Fitness and phenotypic characterization of miltefosineresistant Leishmania major. PLoS Negl Trop Dis. 2015;9:e0003948.

14. Hendrickx S, Beyers J, Mondelaers A, Eberhardt E, Lachaud L, Delputte $P$, et al. Evidence of a drug-specific impact of experimentally selected paromomycin and miltefosine resistance on parasite fitness in Leishmania infantum. J Antimicrob Chemother. 2016;71:1914-21.

15. Hendrickx S, Boulet G, Mondelaers A, Dujardin JC, Rijal S, Lachaud $L$, et al. Experimental selection of paromomycin and miltefosine resistance in intracellular amastigotes of Leishmania donovani and $L$. infantum. Parasitol Res. 2014;113:1875-81.

16. Hendrickx S, Mondelaers A, Eberhardt E, Delputte P, Cos P, Maes L. In vivo selection of paromomycin and miltefosine resistance in Leishmania donovani and L. infantum in a Syrian hamster model. Antimicrob Agents Chemother. 2015;59:4714-8.

17. Quispe Tintaya KW, Ying X, Dedet JP, Rijal S, De Bolle X, Dujardin JC. Antigen genes for molecular epidemiology of leishmaniasis: polymorphism of cysteine proteinase B and surface metalloprotease glycoprotein 63 in the Leishmania donovani complex. J Infect Dis. 2004;189:1035-43.

18. Downing T, Imamura H, Decuypere S, Clark TG, Coombs GH, Cotton $J \mathrm{~A}$, et al. Whole genome sequencing of multiple Leishmania donovani clinical isolates provides insights into population structure and mechanisms of drug resistance. Genome Res. 2011;21:2143-56.

19. Hornillos V, Carrillo E, Rivas L, Amat-Guerri F, Acuna AU. Synthesis of BODIPY-labeled alkylphosphocholines with leishmanicidal activity, as fluorescent analogues of miltefosine. Bioorg Med Chem Lett. 2008;18:6336-9.

20. Vermeersch $M$, da Luz RI, Tote $K$, Timmermans JP, Cos P, Maes L. In vitro susceptibilities of Leishmania donovani promastigote and amastigote stages to antileishmanial reference drugs: practical relevance of stagespecific differences. Antimicrob Agents Chemother. 2009:53:3855-9.

21. Kerstens M, Boulet $G$, Pintelon I, Hellings M, Voeten L, Delputte P, et al. Quantification of Candida albicans by flow cytometry using TO$\mathrm{PRO}((\mathrm{R}))-3$ iodide as a single-stain viability dye. J Microbiol Methods. 2013:92:189-91.

22. Hendrickx S, Leemans A, Mondelaers A, Rijal S, Khanal B, Dujardin JC, et al. Comparative fitness of a parent Leishmania donovani clinical isolate and its experimentally derived paromomycin-resistant strain. PLOS ONE. 2015;10:e0140139.

23. Volf $P$, Volfova $V$. Establishment and maintenance of sand fly colonies. J Vector Ecol. 2011:36(Suppl. 1):S1-9.

24. Dumetz F, Imamura H, Sanders M, Seblova V, Myskova J, Pescher P, et al. Modulation of aneuploidy in Leishmania donovani during adaptation 
to different in vitro and in vivo environments and its impact on gene expression. mBio. 2017;8:e00599-17.

25. Hendrickx S, Guerin PJ, Caljon G, Croft SL, Maes L. Evaluating drug resistance in visceral leishmaniasis: the challenges. Parasitology. 2016;145:453-63.

26. Dorlo TP, Rijal S, Ostyn B, de Vries PJ, Singh R, Bhattarai N, et al. Failure of miltefosine in visceral leishmaniasis is associated with low drug exposure. J Infect Dis. 2014;210:146-53.

27. Ostyn B, Hasker E, Dorlo TP, Rijal S, Sundar S, Dujardin JC, et al. Failure of miltefosine treatment for visceral leishmaniasis in children and men in South-East Asia. PLoS ONE. 2014;9:e100220.

28. Hendrickx SML, Croft SL, Caljon G. The challenges of effective leishmaniasis treatment. In: Sucre AP, editor. Drug resistance in Leishmania parasites. New York: Springer; 2018. p. 193-206.

29. Eberhardt E, Bulte D, Van Bockstal L, Van den Kerkhof M, Cos P, Delputte $P$, et al. Miltefosine enhances the fitness of a non-virulent drug-resistant Leishmania infantum strain. J Antimicrob Chemother. 2018;74:395-406.

30. Mukhopadhyay R, Mukherjee S, Mukherjee B, Naskar K, Mondal D, Decuypere S, et al. Characterisation of antimony-resistant Leishmania donovani isolates: biochemical and biophysical studies and interaction with host cells. Int J Parasitol. 2011;41:1311-21.

31. Shaw CD, Lonchamp J, Downing T, Imamura H, Freeman TM, Cotton $J A$, et al. In vitro selection of miltefosine resistance in promastigotes of Leishmania donovani from Nepal: genomic and metabolomic characterization. Mol Microbiol. 2016;99:1134-48.

32. Seifert K, Matu S, Javier Perez-Victoria F, Castanys S, Gamarro F, Croft SL. Characterisation of Leishmania donovani promastigotes resistant to hexadecylphosphocholine (miltefosine). Int J Antimicrob Agents. 2003;22:380-7.

33. Perez-Victoria FJ, Gamarro F, Ouellette M, Castanys S. Functional cloning of the miltefosine transporter. A novel P-type phospholipid translocase from Leishmania involved in drug resistance. J Biol Chem. 2003;278:49965-71.

34. Hendrickx S, Mondelaers A, Eberhardt E, Lachaud L, Delputte P, Cos $P$, et al. Intracellular amastigote replication may not be required for successful in vitro selection of miltefosine resistance in Leishmania infantum. Parasitol Res. 2015;114:2561-5.

35. Bhandari V, Kulshrestha A, Deep DK, Stark O, Prajapati VK, Ramesh V, et al. Drug susceptibility in Leishmania isolates following miltefosine treatment in cases of visceral leishmaniasis and post kala-azar dermal leishmaniasis. PLoS Negl Trop Dis. 2012;6:e1657.

36. Sterkers Y, Lachaud L, Bourgeois N, Crobu L, Bastien P, Pages M. Novel insights into genome plasticity in Eukaryotes: mosaic aneuploidy in Leishmania. Mol Microbiol. 2012;86:15-23.

37. Vacchina P, Norris-Mullins B, Abengozar MA, Viamontes CG, Sarro J, Stephens MT, et al. Genomic appraisal of the multifactorial basis for in vitro acquisition of miltefosine resistance in Leishmania donovani. Antimicrob Agents Chemother. 2016;60:4089-100.

38. Hendrickx S, Eberhardt E, Mondelaers A, Rijal S, Bhattarai NR, Dujardin $J C$, et al. Lack of correlation between the promastigote back-transformation assay and miltefosine treatment outcome. J Antimicrob Chemother. 2015;70:3023-6.

39. Coelho AC, Boisvert S, Mukherjee A, Leprohon P, Corbeil J, Ouellette M. Multiple mutations in heterogeneous miltefosine-resistant Leishmania major population as determined by whole genome sequencing. PLoS Negl Trop Dis. 2012;6:e1512.

\section{Publisher's Note}

Springer Nature remains neutral with regard to jurisdictional claims in published maps and institutional affiliations.
Ready to submit your research? Choose BMC and benefit from:

- fast, convenient online submission

- thorough peer review by experienced researchers in your field

- rapid publication on acceptance

- support for research data, including large and complex data types

- gold Open Access which fosters wider collaboration and increased citations

- maximum visibility for your research: over $100 \mathrm{M}$ website views per year

At BMC, research is always in progress.

Learn more biomedcentral.com/submissions 